\title{
Topological disorder operators in three-dimensional conformal field theory
}

\author{
Vadim Borokhov, Anton Kapustin and Xinkai Wu \\ California Institute of Technology \\ Pasadena, CA 91125, USA \\ E-mail: borokhov@theory.caltech.edu, kapustin@theory.caltech.edu, \\ xinkaiwu@theory.caltech.edu
}

ABSTRACT: Many abelian gauge theories in three dimensions flow to interacting conformal field theories in the infrared. We define a new class of local operators in these conformal field theories which are not polynomial in the fundamental fields and create topological disorder. They can be regarded as higher-dimensional analogues of twist and winding-state operators in free 2d CFTs. We call them monopole operators for reasons explained in the text. The importance of monopole operators is that in the Higgs phase, they create AbrikosovNielsen-Olesen vortices. We study properties of these operators in three-dimensional QED using large $N_{f}$ expansion. In particular, we show that monopole operators belong to representations of the conformal group whose primaries have dimension of order $N_{f}$. We also show that monopole operators transform non-trivially under the flavor symmetry group, with the precise representation depending on the value of the Chern-Simons coupling.

Keywords: Field Theories in Lower Dimensions, Solitons Monopoles and Instantons, 1/N Expansion, Duality in Gauge Field Theories. 


\section{Contents}

1. Introduction 1

2. Review of three-dimensional QED 2

3. Defining monopole operators 1

3.1 A preliminary definition

3.2 A more precise definition

4. Properties of monopole operators 7

4.1 Radial quantization in the presence of a flux 7

4.2 Quantum numbers of the monopole operators 10

5. Discussion 11

А. Monopole harmonics 13

B. Energy spectrum and Casimir energy for fermions on $S^{2}$

\section{Introduction}

One of the most fascinating problems in quantum field theory is understanding nonperturbative equivalences ("dualities") between superficially very different theories. A classic example is the quantum equivalence of the massive Thirring and sine-Gordon models [1, 2]. More recently, a number of dualities has been conjectured for supersymmetric gauge theories in three and four dimensions. The earliest proposal of this sort is the Sduality of $N=4 d=4$ super-Yang-Mills theory [3, 4, 5]. A decade and a half later, N. Seiberg proposed a dual description for the $4 \mathrm{~d}$ CFT which arises as the infrared limit of $N=1 d=4$ super-QCD [6]. The dual theory is again the infrared limit of an $N=1$ $d=4$ gauge theory. This proposal generated tremendous excitement, and soon many other candidate dualities have been found (see refs. [7, 8 for a review). Later it was realized that many field-theoretic dualities follow from string theory dualities.

Until now, all dualities in dimensions higher than two remain conjectural, and the physical reason for their existence is not completely understood. On the other hand, $2 \mathrm{~d}$ dualities have a rather transparent physical meaning. For example, the sine-Gordon model has topological solitons (kinks), and it can be shown that a certain local operator which creates a kink satisfies the equations of motion of the massive Thirring model [2]. It is believed that many higher-dimensional dualities arise in a similar manner, by "rewriting" the theory in terms of operators which create topological disorder. But it proved very hard to make this idea precise. 
There are several related difficulties that one encounters in dimension higher than two. First of all, interesting higher-dimensional dualities involve gauge theories. This implies that in order to write down an operator describing the dual degrees of freedom, one has to work in an enlarged state space which includes the unphysical degrees of freedom of both the original and the dual gauge fields. It is not known how to construct such an enlarged space. Fortunately, there are non-trivial examples of dualities in three dimensions (so called 3d mirror pairs 9) for some of which the dual theory has a trivial gauge group. In this case one can hope to construct the operators describing the dual degrees of freedom directly in the state space of the original gauge theory.

The second difficulty is that it is hard to construct topological disorder operators in interacting fields theories. For example, it is believed that 3d mirror symmetry arises when one rewrites three-dimensional supersymmetric QED in terms of local operators which create Abrikosov-Nielsen-Olesen vortices [10]. This means that such operators are monopoles. However, it was never clear how to define monopole operators in SUSY QED. A proposal in this direction was made by M. J. Strassler and one of the authors [11], but it was only partially successful.

In this paper we will address the second issue in a simple toy model: three-dimensional QED with $N_{f}$ flavors of fermions. This theory is believed to flow to an interacting conformal fixed point for large enough $N_{f}$ (this is discussed in more detail in the next section). The theory is not supersymmetric and is not expected to possess a simple dual. Nevertheless, we believe it is a useful exercise to define monopole operators in this simple model and learn to work with them. Besides, monopole operators are rather interesting beasts even in the non-supersymmetric case. First of all, these are the first examples of local operators in a three-dimensional CFT which are not polynomial in the fundamental fields. Thus our construction can be regarded as a generalization of the vertex operator construction from free $2 \mathrm{~d}$ CFT to an interacting 3d CFT. Second, we show that because of fermionic zero modes monopole operators transform in a non-trivial representation of the flavor group whose size depends on the Chern-Simons coupling. Monopole operators in supersymmetric QED and their role in mirror symmetry will be discussed in a forthcoming publication.

\section{Review of three-dimensional QED}

The action of three-dimensional QED in the euclidean space is given by

$$
L_{Q E D}=\int d^{3} x\left(\frac{1}{4 e^{2}} F_{\mu \nu} F^{\mu \nu}+\psi_{j}^{\dagger}\left(\sigma \cdot i D_{A}\right) \psi^{j}\right)
$$

Here $A$ is the $\mathrm{U}(1)$ gauge field, $F=d A$ is the field-strength 2-form, $D_{A}$ is the corresponding covariant derivative, and $\psi^{j}$ is a complex two-component spinor. The index $j$ runs from 1 to $N_{f}$.

In three dimensions one can add to the action a Chern-Simons term

$$
L_{C S}=\frac{i k}{4 \pi} \int d^{3} x \epsilon^{\mu \nu \rho} A_{\mu} \partial_{\nu} A_{\rho} .
$$


Such a term breaks parity invariance of the theory. We will assume that the gauge group is compact (i.e. $\mathrm{U}(1)$ rather than $\mathbb{R}$ ). Naively, this requires the Chern-Simons coupling $k$ to be an integer, to avoid global anomalies. The real story is slightly more complicated. When $N_{f}$ is odd, the fermionic path integral is anomalous. The anomaly is the same as the anomaly due to a Chern-Simons term with $k=1 / 2$. Thus cancellation of global anomalies requires

$$
k-\frac{N_{f}}{2} \in \mathbb{Z} .
$$

In particular, for odd $N_{f}$ the Chern-Simons coupling must be non-zero, and parity is broken. This is known as parity anomaly [12].

The gauge coupling $e$ has dimension $m^{1 / 2}$. Thus the theory is super-renormalizable and free in the ultraviolet. In fact, its UV behavior is so good that no renormalization of the lagrangian is required. Contrariwise, 3d QED is strongly coupled in the infrared (perturbative expansion is really an expansion in powers of $e^{2} / p$, where $p$ is the momentum scale). It is natural to assume that the low-energy limit of this theory is a non-trivial CFT, but this has not been conclusively demonstrated. However, the statement holds to all orders in $1 / N_{f}$ expansion [13]-16]. In fact, in the limit $N_{f} \rightarrow \infty$ the infrared theory becomes weakly coupled, and conformal dimensions of all fields can be computed order by order in $1 / N_{f}$. For example, the IR dimension of $\psi^{j}$ is canonical (i.e. the same as the UV dimension) up to corrections of order $1 / N_{f}$.

More interestingly, the IR dimension of $F_{\mu \nu}$ is 2 to all orders in $1 / N_{f}$. To understand why this is the case, consider a current

$$
J^{\mu}=\epsilon^{\mu \nu \rho} F_{\nu \rho} .
$$

It is identically conserved by virtue of the Bianchi identity. A priori, this current could either be a primary field, or a descendant of the primary field. In the UV, the latter possibility is realized, since we can write

$$
J^{\mu}=\partial^{\mu} \sigma,
$$

where $\sigma$ is a free scalar field. The scalar $\sigma$ is usually referred to as the dual photon. It has dimension $1 / 2$ (as befits a free scalar in three dimensions), while $J^{\mu}$ and $F_{\mu \nu}$ have dimension $3 / 2$. On the other hand, in the IR an equation like eq. (2.1) cannot hold. Indeed, eq. (2.1) implies that $F_{\mu \nu}$ obeys the free Maxwell equation, which clashes with the assumption that there are massless charged particles in the infrared. (We assume here that the fermions do not get a mass due to some non-perturbative effect, see a discussion below.) This strongly suggests that in the IR limit $J^{\mu}$ is a primary field. It is well known that in a unitary $3 \mathrm{~d}$ CFT a conserved primary current has dimension 2. Hence the IR dimension of $J^{\mu}$ and $F_{\mu \nu}$ is 2 . This conclusion can also be reached by directly studying the perturbative expansion in powers of $1 / N_{f}$ [16].

Note that the difference between the UV and IR dimensions of $F$ is of order 1, and therefore the IR fixed point is far from the UV fixed point, even in the limit $N_{f} \rightarrow \infty$. In this respect, the situation is very different from the Banks-Zaks-type theories in fourdimensions [17], where the IR dimensions of all operators are very close to their UV dimensions. 
The physics of 3d QED at finite $N_{f}$ remains controversial. The conventional approach is to study the system of Schwinger-Dyson equations truncated in some way and look for symmetry-breaking solutions. For simplicity, let us focus on the case of zero Chern-Simons coupling and even $N_{f}$. It has been claimed that at finite $N_{f}$ flavor symmetry and parity are spontaneously broken by a dynamical mass for the fermions, and the infrared limit is a free theory of photons [18]. The majority of such studies indicate that this happens for $N_{f}$ smaller than a certain critical value of order 6 or 7 (see e.g. [19, 20, 21]). There are also claims that dynamical mass generation takes place for all $N_{f}$ but is exponentially small for large $N_{f}$ and therefore invisible in $1 / N_{f}$ expansion [18, 22, 23]. It must be stressed that the results of such studies depend on the way one truncates an infinite system of SchwingerDyson equations, a procedure which cannot be fully justified. Lattice simulations of $3 \mathrm{~d}$ QED have been inconclusive so far.

In this paper we will be interested in the large $N_{f}$ limit, and therefore the behavior at finite $N_{f}$ will be unimportant. Note also that in the $N=2$ and $N=4$ supersymmetric cases the situation is better, in the sense that one can argue the existence of a non-trivial CFT at the origin of the quantum moduli space for all $N_{f}$.

\section{Defining monopole operators}

\subsection{A preliminary definition}

As mentioned above, three-dimensional QED possesses an interesting conserved current, the dual of the field strength:

$$
J^{\mu}=\frac{1}{4 \pi} \epsilon^{\mu \nu \rho} F_{\nu \rho}
$$

Its conservation is equivalent to the Bianchi identity $d F=0$. The corresponding charge is called the vortex charge, because in the Higgs phase it is carried by the Abrikosov-NielsenOlesen (ANO) vortices. The vortex charge is integral if the gauge field $A$ is a well-defined connection on a $\mathrm{U}(1)$ principal bundle. Loosely speaking, we would like to construct a vortex-creating operator. But in an interacting conformal field theory, it does not make sense to say that an operator is creating a particle. A vortex-creating operator will be defined as an operator with a unit vortex charge. This means that the OPE of such an operator with $J^{\mu}$ has the form

$$
J^{\mu}(x) O(0) \sim \frac{1}{4 \pi} \frac{x^{\mu}}{|x|^{3}} O(0)+\text { less singular terms } .
$$

Such operators can be organized in the representations of the conformal group. In a unitary theory local operators must transform according to lowest-weight representations, i.e. those representations in which the dimension of operators is bounded from below. The operator with the lowest dimension is called a conformal primary. It is standard to label a representation by the spin and dimension of its primary. Our problem can be formulated as follows: determine the spin, dimension, and other quantum numbers of primaries with a given vortex charge. 
In the path integral language, an insertion of an operator with vortex charge $n$ at a point $p$ is equivalent to integrating over gauge fields which have a singularity at $x=p$ such that the magnetic flux through a 2 -sphere surrounding $x=p$ is $n$. To be consistent, one must regard charged matter fields as sections of a non-trivial line bundle on the punctured $\mathbb{R}^{3}$. Thus an insertion of a vortex-creating operator causes a change in the topology of fields near the insertion point. In what follows we will use the terms "vortex-creating operator" and "monopole operator" interchangeably.

This way of defining topological disorder operators is familiar from $2 \mathrm{~d}$ CFT. For example, a twist operator for a free fermion in $2 \mathrm{~d}$ is defined by the condition that the fermion field changes sign as one goes around the insertion point [24. Another example is afforded by the theory of a periodic free boson in $2 \mathrm{~d}$. This theory has winding states, and the corresponding operators create a logarithmic singularity for the boson field. Thus our monopole operators can be regarded as three-dimensional analogues of twist operators or winding-state operators.

In the two-dimensional case one can loosely say that a winding-state operator creates a kink. The precise meaning of this statement is the following. Consider a perturbation of the free boson theory by a periodic potential, say, a sine-Gordon potential. The resulting massive theory has multiple vacua and topological excitations (kinks) interpolating between neighboring vacua. The operator which carries winding number one has non-zero matrix elements between the vacuum and the one-kink state.

Similarly, one can loosely say that a monopole operator creates an ANO vortex. To make this statement precise, one has to go to the Higgs phase (for example, by adding charged scalars with an appropriate potential). In the Higgs phase, the magnetic flux emanating from the insertion point of the monopole operator is squeezed into a thin tube. This tube is the world-line of a vortex.

\subsection{A more precise definition}

The definition of monopole operators given above is not yet complete. In effect, we have defined an insertion of a monopole operator by requiring that the gauge field strength have a particular singularity at the insertion point. However, we did not specify the behavior of the matter fields near the insertion point. In fact, we expect that there are many operators which carry the same vortex charge, and they differ precisely by the behavior of fields at the insertion point. Another difficulty is that the IR theory is strongly coupled, and it seems hard to compute correlators involving monopole operators.

The first difficulty can be circumvented using radial quantization. It is a general feature of CFT in any dimension that local operators are in one-to-one correspondence with states in the Hilbert space of the radially quantized theory. This follows from the fact that one can use a conformal transformation to map an insertion point of an operator to infinity. In this way one trades a local operator for an incoming or out-going state. In the case of monopole operators, such a mapping takes an operator with vortex charge $n$ to a state on $\mathbf{S}^{2} \times \mathbb{R}$ with a magnetic flux $n$ through $\mathbf{S}^{2}$. Here $\mathbb{R}$ is regarded as the time direction. Classifying states of a CFT on $\mathbf{S}^{2} \times \mathbb{R}$ with a given vortex charge is certainly a well-defined problem. Furthermore, the radially quantized picture is the most convenient 
one for computing correlators which involve two monopole operators with opposite vortex charges and an arbitrary number of ordinary operators. By mapping the insertion of a monopole operator to an in-going state and the insertion of an anti-monopole operator to an out-going state, one reduces the problem to computing a particular matrix element of a product of several ordinary operators. A particularly important special case is the three-point function which involves a monopole operator, an anti-monopole operator, and a conserved current. Knowledge of such correlators allows one to read off the quantum numbers of a monopole operator. For example, in order to determine the dimension of an operator, one has to compute the expectation value of the stress-energy tensor in the corresponding state. This approach is familiar from $2 \mathrm{~d}$ CFT, where it is used to compute the quantum numbers of twist operators (see e.g. ref. [24]).

Of course, if one desires to compute four-point functions of monopole operators, mapping two of the insertion points to infinity does not help very much. In the case of $2 \mathrm{~d}$ $\mathrm{CFT}$, one has to use tricks special to the theory in question in order to compute four-point functions of topological disorder operators. In this paper, we will be content with studying the OPE of monopole operators with conserved currents, and leave the study of four-point functions to future work.

The second difficulty can be avoided by working in the large $N_{f}$ limit. It is a general feature of this limit that the gauge field does not fluctuate, and can be treated classically [13. 14, 16. This can be seen as follows. The infrared limit in 3d QED is simply the limit $e \rightarrow \infty$. This is literally true, because no renormalization of the lagrangian is required. Thus one can simply drop the kinetic term for the gauge field. Integrating out the fermions then gives an effective action for the gauge field of order $N_{f}$. For example, when expanded around a trivial background, this action looks like

$$
N_{f} \int\left(F_{\mu \nu} \square^{-1 / 2} F^{\mu \nu}+\text { higher-order terms }\right) d^{3} x .
$$

Thus the effective Planck constant is of order $1 / N_{f}$, and in the large $N_{f}$ limit the size of gauge-field fluctuations is order $1 / N_{f}$. Moreover, if we absorb a factor of $N_{f}^{1 / 2}$ into $F$, we see that self-interactions of $F$ are suppressed in the large $N_{f}$ limit. In other words, $N_{f}^{1 / 2} F$ is a gaussian field in the large $N_{f}$ limit. It is this line of reasoning that allows one to show that the infrared CFT is weakly coupled in the large $N_{f}$ limit. The argument also applies to CFT on $\mathbf{S}^{2} \times \mathbb{R}$ with a flux. Thus we can regard the gauge field as a classical background.

It is very plausible that the saddle point of the effective action for $F$ on $\mathbf{S}^{2} \times \mathbb{R}$ is rotationally symmetric. Therefore we can assume that the classical background is simply a constant magnetic flux on $\mathbf{S}^{2}$.

The above discussion reduced our problem to studying free fermions on $\mathbf{S}^{2} \times \mathbb{R}$ in the presence of a constant magnetic flux. This is almost a textbook problem, and everything of interest can be computed. For example, finding the dimension of a monopole operator is equivalent to computing the Casimir energy of free fermions on $\mathbf{S}^{2}$ with a flux. It is a priori clear that this energy scales like $N_{f}$. There are corrections to this result, which can be computed by taking into account the fluctuations of the gauge field. However, such effects are suppressed by powers of $1 / N_{f}$. 
The above discussion contains a gap as regards gauge invariance of monopole operators. Gauge-invariance of a local operator is equivalent to gauge-invariance of the corresponding state in the radially quantized picture. In other words, the state must satisfy the Gauss' law. Gauss' law in QED on $\mathbf{S}^{2} \times \mathbb{R}$ comes from varying the action with respect to the "time-like" component of the gauge field $A$. In the limit $e \rightarrow \infty$ it simply reads

$$
\rho(x)|\Phi\rangle=0
$$

where

$$
\rho(x)=\sum_{j} \psi_{j}^{\dagger}(x) \psi^{j}(x)
$$

is the electric charge density operator. In particular, the total electric charge of a gaugeinvariant state must be zero. The latter is a standard fact about gauge theory on a compact space, valid irrespective of the value of $e$. The definition of the electric charge operator involves normal-ordering ambiguities, which will be dealt with below. Note also that the inclusion of the Chern-Simons term in the action modifies the Gauss' law constraint into

$$
\left(\rho+\frac{k}{4 \pi} \epsilon^{i j} F_{i j}\right)|\Phi\rangle=0 .
$$

In particular, the total electric charge of the matter modes must be equal to $-k$ times the vortex charge. In this way (and only in this way) the Chern-Simons term will affect the physics at large $N_{f}$.

\section{Properties of monopole operators}

\subsection{Radial quantization in the presence of a flux}

As explained in the previous section, at large $N_{f}$ all properties of monopole operators can be deduced from the properties of free fermions on $\mathbf{S}^{2} \times \mathbb{R}$ in a constant background magnetic flux. In this subsection we summarize the physics of this system, with detailed derivations relegated to appendix A.

The spectrum of the Dirac hamiltonian on $\mathbf{S}^{2} \times \mathbb{R}$ with $n$ units of magnetic flux is given by

$$
E_{p}= \pm \sqrt{p^{2}+p|n|}, \quad p=0,1,2, \ldots .
$$

The degeneracy of the $p$-th eigenvalue is $2 j_{p}+1$, where

$$
j_{p}=\frac{1}{2}(|n|-1)+p .
$$

These $2 j_{p}+1$ states transform as an irreducible representation of the rotation group $\mathrm{SU}(2)_{\text {rot }}$.

The presence of $n$ states with zero energy is particularly important. The existence of at least $n$ zero modes is dictated by the Atiyah-Singer index theorem applied to the Dirac operator on $\mathbf{S}^{2}$ coupled to the magnetic field.

In the case of a unit magnetic flux $(|n|=1)$, we have a single fermionic zero mode with zero spin. Thus a spinor is converted into a scalar due to the non-trivial topology of the magnetic monopole. This scalar-spinor transmutation is well known in other contexts; 
in particular it plays an important role in the conjectured S-duality of $N=4 d=4$ super-Yang-Mills. For general $n$, the fermionic zero modes transform in an irreducible representation of $\mathrm{SU}(2)_{\text {rot }}$ with spin $j=(|n|-1) / 2$. We will discuss in detail the case when $n= \pm 1$, and then comment on the higher- $n$ case.

Let us denote the fermionic annihilation operators by $c_{p m}^{i}$, where $i=1, \ldots, N_{f}$ is the flavor index, $p=1,2, \ldots$, labels the energy eigenspaces as above, and $m=-j_{p},-j_{p}+$ $1, \ldots, j_{p}$, labels the states within the $p$-th energy eigenspace. The fermion annihilation operators corresponding to $p=0$ will be denoted simply by $c_{0}^{i}$. The Hilbert space of the theory is the tensor product of the Hilbert space of zero modes and the Hilbert space of all other modes. The latter is simply a fermionic Fock space with a unique vacuum $|v a c\rangle_{+}$ which satisfies

$$
c_{p m}^{i}|v a c\rangle_{+}=0, \quad p>0, \forall i, m .
$$

This vacuum state is rotationally invariant.

The Hilbert space of zero modes is also a Fock space of dimension $2^{N_{f}}$, with the vacuum vector which we denote $|v a c\rangle_{0}$. It is spanned by the vectors

$$
|v a c\rangle_{0}, \quad c_{0}^{i \dagger}|v a c\rangle_{0}, \quad c_{0}^{i_{1} \dagger} c_{0}^{i_{2} \dagger}|v a c\rangle_{0}, \quad \ldots, \quad c_{0}^{i_{1} \dagger} c_{0}^{i_{2} \dagger} \ldots c_{0}^{i_{N_{f}} \dagger}|v a c\rangle_{0}
$$

All these states are degenerate in energy, and none is a preferred vacuum. Since the zero modes have spin zero, all the ground states are rotationally invariant. We conclude that

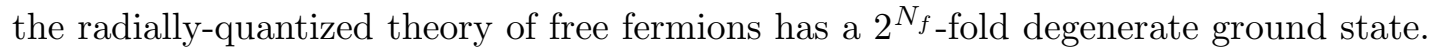

However, we still need to impose the Gauss' law. The charge density operator receives contributions from both zero and non-zero modes. The part due to non-zero modes can be defined using the obvious normal-ordering prescription. If we put all non-zero modes in the vacuum state, then the charge density due to non-zero modes vanishes. It remains to analyze the contribution from zero modes. Naively, it seems that the Fock vacuum must be assigned zero electric charge. If this were the case, then the states obtained by acting on the vacuum with zero mode creation operators would have positive charge, and therefore would not be gauge-invariant. But because of normal-ordering ambiguities, the situation is more interesting.

As stressed above, the Fock vacuum for the zero modes is not that special. The completely filled state appears to be an equally good candidate for a state with vanishing electric charge. The two just differ by a change in the normal ordering prescription. A statement which is independent of the normal-ordering prescription is that the electric charge of the filled state exceeds the charge of the vacuum by $N_{f}$. If one wants to be "democratic", one has to assign charge $-\frac{1}{2} N_{f}$ to the vacuum and charge $\frac{1}{2} N_{f}$ to the filled state. A similar symmetric charge assignment has been advocated by Jackiw and Rebbi in their pioneering study of fermions bound to solitons, on the grounds on charge-conjugation symmetry [25].

The precise argument for the symmetric charge assignment goes as follows. Charge conjugation maps a monopole to an anti-monopole and by itself does not tell us anything. But CP transformation maps a monopole to itself. If we want to quantize in a CP-invariant manner, we must assign opposite electric charges to states related by CP. Since CP takes 
annihilation operators into creation operators, the filled state and the vacuum are related by $\mathrm{CP}$, and their electric charges must be opposite.

The invocation of CP invariance assumes that the theory we started with is CPinvariant. This means that the symmetric charge assignment is valid for a vanishing ChernSimons coupling. But we know that turning on the Chern-Simons coupling $k$ is equivalent to shifting the electric charge by $k$ times the vortex charge. Therefore we conclude that in the presence of the Chern-Simons coupling the Fock vacuum has electric charge

$$
-\frac{N_{f}}{2}+k,
$$

while the filled state has charge

$$
\frac{N_{f}}{2}+k
$$

Note that because of the parity anomaly, the electric charge is all always integervalued, whether $N_{f}$ is even or odd. This a manifestation of the close relationship between the existence of parity anomaly and the induced vacuum charge [26].

Now we can analyze the consequences of the Gauss' law constraint. If all non-zero modes are in their ground state, the constraint simply says that the total electric charge of the state must be zero. For $k=0$ this implies that a physical state is obtained by acting with $N_{f} / 2$ zero modes on the vacuum. The number of such states is

$$
\left(\begin{array}{c}
N_{f} \\
\frac{1}{2} N_{f}
\end{array}\right)
$$

and they transform as an anti-symmetric tensor of $\mathrm{SU}\left(N_{f}\right)$ with $N_{f} / 2$ indices. Note that cancellation of global anomalies requires $N_{f}$ to be even when $k=0$, so this result makes sense. For $k$ between $-N_{f} / 2$ and $N_{f} / 2$ the physical states are obtained by acting with $N_{f} / 2-k$ zero modes on the vacuum. The corresponding states transform as an antisymmetric tensor of $\mathrm{SU}\left(N_{f}\right)$ with $N_{f} / 2-k$ indices. Again global anomaly cancellation ensures that $N_{f} / 2-k$ is an integer. For $|k|>N_{f} / 2$ there are no gauge-invariant states with unit vortex charge and all non-zero modes in their ground state.

If one does not assume that positive-energy modes are in their ground state, then one can construct many other states which satisfy the Gauss' law and have unit vortex charge. However, such states will have higher energy than the ones discussed above.

Now let us consider the more complicated case of $n=2$. For simplicity we will set the Chern-Simons coupling to zero and take $N_{f}$ to be even. In the case $n=2$ each fermion has two zero modes which transform as a spin-1/2 representation of $\mathrm{SU}(2)_{\text {rot }}$. Reasoning based on CP-invariance tell us that the Fock vacuum has electric charge $-N_{f}$. Physical states must have zero electric charge and are obtained by acting with $N_{f}$ zero modes (out of a total number of $2 N_{f}$ ) on the vacuum. But physical states must also be annihilated by the electric charge density operator. This is not automatic anymore, because the fermionic zero modes are not rotationally invariant. A short computation shows that the electric charge density operator for the zero modes $\rho_{0}(x)$ has a piece which transforms as a singlet of $\mathrm{SU}(2)_{\text {rot }}$ and a piece which transforms as a triplet of $\mathrm{SU}(2)_{\text {rot }}$. The former is simply 
the average of $\rho_{0}(x)$ over the sphere and is proportional to the total electric charge. The spin-triplet piece of $\rho_{0}(x)$ is proportional to the total spin, simply because this is the only spin-triplet one can make out of two spin-1/2 fermions. Thus the Gauss' law constraint is equivalent to the requirement that the total electric charge as well as the total spin be zero.

For example, for $N_{f}=2$, there are six states with zero total electric charge, which are obtained by acting on the Fock vacuum with two zero modes out of the available four. Three of these states transform as a vector of $\mathrm{SU}(2)$ rot and as singlets of the flavor group $\mathrm{SU}(2)_{f}$ and do not satisfy the Gauss' law constraint. The remaining three transform as singlets of $\mathrm{SU}(2)_{\text {rot }}$ and as a triplet of $\mathrm{SU}(2)_{f}$. These three states are gauge-invariant. Note that in this case gauge-invariant states transform as an irreducible representation of the flavor group. For $N_{f}>2$ this is no longer true, as one can easily check.

\subsection{Quantum numbers of the monopole operators}

In this section we determine the quantum numbers of the simplest monopole operators, the ones with the lowest conformal dimension for a given vortex charge. On general grounds, such an operator lives in a lowest-weight representation of the conformal group, and its conformal dimension is defined as the conformal dimension of the lowest-weight vector, or, if we pass to the radially quantized picture, as the energy of the corresponding state.

Let us begin with the case $n=1$. As explained above, gauge-invariant states with lowest energy are obtained by putting all non-zero modes in their ground states and acting by $N_{f} / 2-k$ zero mode creation operators on the vacuum. Obviously such states transform as an anti-symmetric representation of $\mathrm{SU}\left(N_{f}\right)$ with $N_{f} / 2-k$ indices. It is interesting to note that the usual gauge-invariant operators which are polynomials in the fundamental fields transform trivially under the center of $\mathrm{SU}\left(N_{f}\right)$. Indeed, free fermions have flavor symmetry group $\mathrm{U}\left(N_{f}\right)$, and since we are gauging its $\mathrm{U}(1)$ subgroup, the flavor symmetry of QED appears to be $\mathrm{U}\left(N_{f}\right) / \mathrm{U}(1)=P \mathrm{U}\left(N_{f}\right)=\mathrm{SU}\left(N_{f}\right) / \mathbb{Z}_{N_{f}}$. But monopole operators transform non-trivially under $\mathbb{Z}_{N_{f}}$ (except for $k= \pm N_{f} / 2$ ). A very similar effect occurs in $N=2 d=4$ super-QCD, where all perturbative states transform as tensor representations of the flavor group $\mathrm{SO}\left(2 N_{f}\right)$, while magnetically charge states transform as spinors 27.

Other quantum numbers of interest are spin and conformal dimension. Since the Fock vacuum and the zero modes are rotationally invariant, the spin of our monopole operator is zero. The dimension is proportional to the energy of the state. As usual, the definition of the energy is plagued by ordering ambiguities. However, we have a simple cure: we can normalize the energy by requiring that the unit operator have zero dimension. This means that the energy of the ground state on $\mathbf{S}^{2}$ with zero magnetic flux is defined to be zero. The energy of any other state can be defined by introducing a UV regulator, subtracting the regularized energy of the state corresponding to the unit operator, and then removing the regulator. This procedure gives a finite answer, which is not sensitive to the precise choice of the regulator, provided the regulator preserves the symmetries of the problem. 
In order to make precise the relation between the Casimir energy and the dimension, recall that the OPE of a spin-zero primary field and the stress-energy tensor reads:

$$
T_{\mu \nu}(x) O(y) \sim \frac{h}{8 \pi}\left(\frac{\partial}{\partial x^{\mu}} \frac{\partial}{\partial x^{\nu}} \frac{1}{|x-y|}\right) O(y)+\ldots,
$$

where $h$ is the conformal dimension. If the stress-energy tensor of free fermions is defined by

$$
T_{\mu \nu}=-\frac{i}{4} \bar{\psi}\left(\gamma_{\mu} \mathcal{D}_{\nu}+\gamma_{\nu} \mathcal{D}_{\mu}\right) \psi+\frac{i}{4}\left(\mathcal{D}_{\nu} \bar{\psi} \gamma_{\mu}+\mathcal{D}_{\mu} \bar{\psi} \gamma_{\nu}\right) \psi-g_{\mu \nu} \mathcal{L}
$$

then $h_{\psi}=h_{\bar{\psi}}=1$, the standard normalization. This implies that in the radially-quantized picture the expectation value of the stress-energy tensor in the state $|O\rangle$ is given by

$$
\left\langle T_{\mu \nu} d x^{\mu} \otimes d x^{\nu}\right\rangle_{O}=\frac{h}{4 \pi}\left(d \tau^{2}-\frac{1}{2}\left(d \theta^{2}+\sin \theta^{2} d \phi^{2}\right)\right)
$$

Thus $h$ is simply the energy of $|O\rangle$ with respect to the Killing vector $\frac{\partial}{\partial \tau}$. In our case, this means that the conformal dimension of the monopole operator is the Casimir energy of $N_{f}$ free fermions on $\mathbf{S}^{2}$ with a magnetic flux. This Casimir energy for any $n$ is computed in the appendix $\mathrm{B}$. For $n=1$ the result is

$$
h_{1}=N_{f} \cdot 0.265 \ldots
$$

By charge-conjugation symmetry, monopole operator with $n=-1$ has the same conformal dimension and spin and transforms in the conjugate representation of the flavor group $\mathrm{SU}\left(N_{f}\right)$.

It is easy to extend the discussion to $n= \pm 2$. As explained in the previous section, Gauss' law constraint is equivalent to the requirement of zero spin and zero electric charge. The states with zero electric charge are obtained by acting with $N_{f}$ zero modes (out of total number of $2 N_{f}$ zero modes) on the Fock vacuum. These states transform as an anti-symmetric tensor of $\mathrm{SU}\left(2 N_{f}\right)$ with $N_{f}$ indices. Gauge-invariant states are obtained by decomposing this representation with respect to the $\mathrm{SU}(2)_{\text {rot }} \times \mathrm{SU}\left(N_{f}\right)$ subgroup

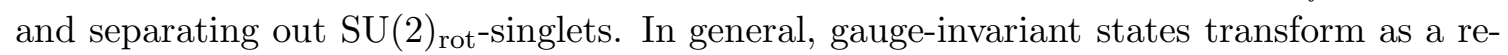
ducible representation of $\mathrm{SU}\left(N_{f}\right)$. One can easily show that the dimension of this reducible representation is

$$
\left(\begin{array}{c}
\frac{1}{2} N_{f}^{2}+N_{f}-1 \\
\frac{1}{2} N_{f}
\end{array}\right)
$$

The conformal dimension of the corresponding monopole operators is the Casimir energy of free fermions in a background magnetic field. Numerically, it is given by

$$
h_{2}=N_{f} \cdot 0.673 \ldots
$$

It is interesting to note that $2 h_{1}<h_{2}$ (at least for large $N_{f}$ ). Therefore the OPE of two monopole operators with $n=1$ and the lowest conformal dimension contains only terms with positive powers of $\left|x_{1}-x_{2}\right|$. 


\section{Discussion}

In this paper we have constructed local operators in an interacting 3d CFT which carry vortex charge and therefore create Abrikosov-Nielsen-Olesen vortices in the Higgs phase. We have shown that for large $N_{f}$ such operators have conformal dimensions of order $N_{f}$. For the case of unit vortex charge, we showed that the operator with the lowest possible dimension has zero spin and transforms in a non-trivial representation of the flavor group. An important tool in this analysis is $1 / N_{f}$ expansion.

The idea that vortex-creation operators can be studied in the large $N_{f}$ limit has been previously proposed in ref. [11]. The approach taken there was to integrate out the matter fields, and then perform a duality transformation on the effective action for the gauge field. Then the vortex-creation operator is defined as the exponential of the dual photon. One drawback of this approach is that it is easy to miss fermionic zero modes, and consequently to misidentify the quantum numbers of the vortex-creating operator. It is preferable to keep the matter fields, and to identify a vortex-creating operator by the property that its insertion causes a change in the topology of the gauge field. As we have seen above, this definition can be made concrete by using radial quantization and large $N_{f}$ expansion.

Our main motivation for studying vortex-creation operators was the hope that this would enable us to give a constructive proof of $3 \mathrm{~d}$ mirror symmetry. It is straightforward to apply the methods of this paper to $3 \mathrm{~d}$ gauge theories with $N=2$ and $N=4$ supersymmetry. The results and their implications for mirror symmetry will be reported in a forthcoming publication.

It is natural to wonder if our approach to the construction of topological disorder operators has an analogue in four dimensions. In three dimensions, we defined the vortex charge of a local operator as the first Chern class of the gauge bundle evaluated on an $\mathbf{S}^{2}$ surrounding the insertion point. In four dimensions, we have $\mathbf{S}^{3}$ instead of $\mathbf{S}^{2}$, and since characteristic classes of vector bundles are even-dimensional, it appears impossible to define a similar topological charge for local operators. On the other hand, a B-field on an $\mathbf{S}^{3}$ can have non-trivial topology, since its field-strength is a 3-form. Thus, if there were an interacting 4d CFT involving a B-field, one could define local operators which create topological disorder. In order for this to work, the field-strength 3-form must have dimension 3, so that its dual is a conserved primary current. Note that in the theory of a free B-field, the field-strength has dimension 2. In this case the dual current, although conserved, is not a primary, but a gradient of a free scalar. Thus in order to define a conformally-invariant topological charge, the 4d CFT must be interacting. Unfortunately, no such theory is known at present. Perhaps there exists a duality-symmetric reformulation of $N=4 d=4$ super-Yang-Mills which involves B-fields, and in which both W-bosons and dual W-bosons are described by topological disorder operators.

After the first version of this paper was posted on the arXive, we learned that topological disorder operators in $3 \mathrm{~d}$ have been previously considered by G. Murthy and S. Sachdev [28]. The model considered there was the $\mathbb{C P}^{N}$ model in three dimensions. This theory is not renormalizable and requires an ultraviolet cut-off. It has a critical point separating the ordered phase, where the sigma-model field has an expectation value, and the disordered 
phase, where the correlators decay exponentially. Unlike in 3d QED, there are dynamical topological defects in the $\mathbb{C P}^{N}$ model (so-called hedgehogs). But at the critical point and in the limit of large- $N$ their density vanishes, and the situation becomes very similar to that in 3d QED. In particular, the critical exponents computed in ref. [28 can be interpreted as scaling dimensions of hedgehog operators. It is interesting to note that the approach to computing these scaling dimensions taken by Murthy and Sachdev is rather different from ours. Instead of evaluating the expectation value of the stress-energy tensor in the presence of topological disorder operators, they in essence compute the 2-point function of these operators. This is somewhat obscured by the fact that Murthy and Sachdev map both operator insertions to infinity. As a result, the distance dependence of the 2-point function is traded for an anomalous dependence of the partition function on the ratio of the ultraviolet cut-off $\Lambda$ and the infrared cut-off $\Delta$. This method could be used in $3 \mathrm{~d}$ QED as well. In fact, M. J. Strassler and one of the authors of the present paper (A.K.) have contemplated such a route to computing scaling dimensions of monopole operators in 3d QED and SQED, but were discouraged by apparent technical difficulties. It would be interesting to rederive the results of the present paper using the approach of ref. [28.

\section{A. Monopole harmonics}

To solve for the energy spectrum of free fermions on $\mathbf{S}^{2}$ with a magnetic flux, we will use the fact that this system is related by a conformal transformation to the Dirac equation on $\mathbb{R}^{3}$ in the monopole background. This allows us to use the machinery of "monopole harmonics" developed by Wu and Yang [29].

The three-dimensional Dirac operator on flat $\mathbb{R}^{3}$ is given by

$$
i D=-\vec{\sigma} \cdot \vec{\pi},
$$

where $\sigma_{x}, \sigma_{y}$, and $\sigma_{z}$ are the Pauli matrices, and $\vec{\pi}=\vec{p}+\vec{A}$, with $\vec{p}$ being the momentum operator. Following ref. [29], let us define the generalized orbital angular momentum operator as

$$
\vec{L}=\vec{r} \times \vec{\pi}-\frac{q \vec{r}}{r}
$$

with $q=-e g=n / 2$. It is straightforward to check that $\vec{L}$ defined this way satisfies the angular momentum algebra:

$$
\begin{aligned}
& {\left[L_{j}, x_{k}\right]=i \epsilon_{j k m} x_{m}} \\
& {\left[L_{j}, \pi_{k}\right]=i \epsilon_{j k m} \pi_{m},} \\
& {\left[L_{j}, L_{k}\right]=i \epsilon_{j k m} L_{m} .}
\end{aligned}
$$

We define the total angular momentum as

$$
\vec{J}=\vec{L}+\frac{\vec{\sigma}}{2}
$$

We can take $r, \vec{L}^{2}, \vec{J}^{2}$, and $J_{z}$ as a complete set of observables (it is easy to check that they commute and are all self-adjoint with respect to the usual inner product). It can be 
checked that $\left[\vec{J}, i D=0\right.$, but $\left[\vec{L}^{2}, i D\right] \neq 0$. However, this is good enough, because as we will see later, to find the eigenvalues of $i D$ we only need to diagonalize an operator in a two-dimensional space.

The monopole harmonics $Y_{q, l, m}(\theta, \phi)$ constructed in ref. [29] satisfy

$$
\begin{array}{r}
\vec{L}^{2} Y_{q, l, m}=l(l+1) Y_{q, l, m}, \quad L_{z} Y_{q, l, m}=m Y_{q, l, m}, \\
l=|q|,|q|+1,|q|+2, \ldots, \quad m=-l, \ldots, l .
\end{array}
$$

The simultaneous eigenfunctions of $\left\{\vec{L}^{2}, \vec{J}^{2}, J_{z}\right\}$ will be denoted by $\phi_{l j m_{j}}$ and are given by

$$
\begin{aligned}
& \phi_{l j m_{j}}=\left(\begin{array}{c}
\sqrt{\frac{l+m+1}{2 l+1}} Y_{q, l, m} \\
\sqrt{\frac{l-m}{2 l+1}} Y_{q, l, m+1}
\end{array}\right) \quad \text { for } \quad j=l+\frac{1}{2}\left(m_{j}=m+\frac{1}{2}\right), \\
& \phi_{l j m_{j}}=\left(\begin{array}{c}
-\sqrt{\frac{l-m}{2 l+1}} Y_{q, l, m} \\
\sqrt{\frac{l+m+1}{2 l+1}} Y_{q, l, m+1}
\end{array}\right) \quad \text { for } \quad j=l-\frac{1}{2}\left(l \neq 0, m_{j}=m+\frac{1}{2}\right) .
\end{aligned}
$$

They satisfy

$$
\begin{aligned}
\vec{L}^{2} \phi_{l j m_{j}} & =l(l+1) \phi_{l j m_{j}}, \\
\vec{J}^{2} \phi_{l j m_{j}} & =j(j+1) \phi_{l j m_{j}}, \\
J_{z} \phi_{l j m_{j}} & =m_{j} \phi_{l j m_{j}} .
\end{aligned}
$$

We can summarize the possible value of $l, j, m_{j}$ as follows:

- $j=|q|-\frac{1}{2}, \quad|q|+\frac{1}{2}, \quad|q|+\frac{3}{2}, \quad|q|+\frac{5}{2}, \ldots$

$$
\text { (for } q=0, j=|q|-\frac{1}{2} \text { is not allowed); }
$$

- if $j=|q|-\frac{1}{2}, \quad$ then $\quad l=j+\frac{1}{2}=|q|, \quad$ otherwise $\quad l=j \pm \frac{1}{2}$;

- $m_{j}=-j,-(j-1), \ldots, j-1, j$.

Any wave-function can be expanded as

$$
\psi(\vec{r})=\sum_{l, j, m_{j}} R_{l j m_{j}}(r) \phi_{l j m_{j}}(\theta, \phi) .
$$

Note that while $\phi_{l j m_{j}}$ is a two-component spinor, $R_{l j m_{j}}$ is just a scalar.

Now we may write $i D$ in terms of the angular momenta. Define $\sigma_{r}$ as

$$
\sigma_{r}=\frac{\vec{\sigma} \cdot \vec{r}}{r} .
$$

One can show that

$$
\sigma_{r}(i D)=i \frac{\partial}{\partial r}-i \frac{1}{r} \vec{\sigma} \cdot \vec{L}-i q \frac{\sigma_{r}}{r}
$$


where we made use of the fact that

$$
(\vec{\sigma} \cdot \vec{G})(\vec{\sigma} \cdot \vec{K})=\vec{G} \cdot \vec{K}+i \vec{\sigma} \cdot(\vec{G} \times \vec{K})
$$

for any $\vec{G}$ and $\vec{K}$ that commute with $\vec{\sigma}$. Now using the fact that ${\sigma_{r}}^{2}=1$, we have

$$
\begin{aligned}
i D & =\sigma_{r} \sigma_{r}(i D)=i \sigma_{r} \frac{\partial}{\partial r}-i \frac{\sigma_{r}}{r} \vec{\sigma} \cdot \vec{L}-i q \frac{1}{r} \\
& =i \sigma_{r} \frac{\partial}{\partial r}-i \frac{\sigma_{r}}{r}\left(\vec{J}^{2}-\vec{L}^{2}-\frac{3}{4}\right)-i q \frac{1}{r} .
\end{aligned}
$$

Thus the Dirac lagrangian on $\mathbb{R}^{3}$ in the presence of a monopole can be written as

$$
\mathcal{L}_{R^{3}}=\frac{i}{r} \bar{\psi} \sigma_{r}\left(r \frac{\partial}{\partial r}-\left(\vec{J}^{2}-\vec{L}^{2}-\frac{3}{4}\right)-q \sigma_{r}\right) \psi .
$$

Setting $r=e^{\tau}$ and performing a Weyl rescaling

$$
g_{\mu \nu} \rightarrow e^{-2 \tau} g_{\mu \nu}, \quad \psi, \bar{\psi} \rightarrow e^{-\tau} \psi, e^{-\tau} \bar{\psi}, \quad \vec{A} \rightarrow \vec{A},
$$

we obtain the lagrangian on $\mathbf{S}^{2} \times \mathbb{R}$ :

$$
\mathcal{L}_{\mathbf{S}^{2} \times \mathbb{R}}=i \bar{\psi} \sigma_{r}\left(\frac{\partial}{\partial \tau}-\left(\vec{J}^{2}-\vec{L}^{2}+\frac{1}{4}\right)-q \sigma_{r}\right) \psi .
$$

Note that the norm

$$
\int_{\mathbf{S}^{2}} r^{2} d \Omega \bar{\psi} \sigma_{r} \psi
$$

on $\mathbb{R}^{3}$ is transformed to the norm

$$
\int_{\mathbf{S}^{2}} \bar{\psi} \sigma_{r} \psi
$$

on $\mathbf{S}^{2} \times \mathbb{R}$.

Taking into account the above results, the euclidean equation of motion for $\psi$ is

$$
\frac{d R_{l j m_{j}}(\tau)}{d \tau}-\left(j(j+1)-l(l+1)+\frac{1}{4}\right) R_{l j m_{j}}(\tau)-\sum_{l^{\prime} j^{\prime} m_{j}^{\prime}} q R_{l^{\prime} j^{\prime} m_{j}^{\prime}}(\tau)\left\langle l j m_{j}\left|\sigma_{r}\right| l^{\prime} j^{\prime} m_{j}^{\prime}\right\rangle=0,
$$

where we have used $\left\langle l j m_{j}\left|\sigma_{r}\right| l^{\prime} j^{\prime} m_{j}^{\prime}\right\rangle$ to denote $\int d \Omega \phi_{l j m_{j}}^{\dagger} \sigma_{r} \phi_{l^{\prime} j^{\prime} m_{j}^{\prime}}$.

Now the identity $\left[\vec{J}, \sigma_{r}\right]=0$ tells us that

$$
\left\langle l j m_{j}\left|\sigma_{r}\right| l^{\prime} j^{\prime} m_{j}^{\prime}\right\rangle=\delta_{j j^{\prime}} \delta_{m_{j} m_{j}^{\prime}}\left\langle l j m_{j}\left|\sigma_{r}\right| l^{\prime} j m_{j}\right\rangle
$$

and thus the eigenvalue equation becomes, for any given $j, m_{j}$,

$$
\frac{d R_{l j m_{j}}(\tau)}{d \tau}-\left(j(j+1)-l(l+1)+\frac{1}{4}\right) R_{l j m_{j}}(\tau)-\sum_{l^{\prime}} q R_{l^{\prime} j m_{j}}(\tau)\left\langle l j m_{j}\left|\sigma_{r}\right| l^{\prime} j m_{j}\right\rangle=0 .
$$

Let us suppress the $j, m_{j}$ indices, and denote $R_{\left(l=j-\frac{1}{2}\right) j m_{j}}$ by $R^{a},\left|l=j-1 / 2, j m_{j}\right\rangle$ by $|a\rangle$, $R_{\left(l=j+\frac{1}{2}\right) j m_{j}}$ by $R^{b},\left|l=j+1 / 2, j m_{j}\right\rangle$ by $|b\rangle,\left\langle a\left|\sigma_{r}\right| a\right\rangle$ by $\sigma_{a a},\left\langle a\left|\sigma_{r}\right| b\right\rangle$ by $\sigma_{a b},\left\langle b\left|\sigma_{r}\right| a\right\rangle$ by $\sigma_{b a}$, 
and $\left\langle b\left|\sigma_{r}\right| b\right\rangle$ by $\sigma_{b b}$. Then for any given $j, m_{j}$, we have two coupled first-order differential equations:

$$
\begin{aligned}
& \frac{d R^{a}(\tau)}{d \tau}=\left(j+\frac{1}{2}\right) R^{a}(\tau)+q\left(\sigma_{a a} R^{a}(\tau)+\sigma_{a b} R^{b}(\tau)\right), \\
& \frac{d R^{b}(\tau)}{d \tau}=-\left(j+\frac{1}{2}\right) R^{b}(\tau)+q\left(\sigma_{b b} R^{b}(\tau)+\sigma_{b a} R^{a}(\tau)\right) .
\end{aligned}
$$

A straightforward calculation of the matrix elements $\sigma_{a a}, \sigma_{a b}$, and $\sigma_{b b}$ gives

$$
\sigma_{a a}=\frac{-q}{j+\frac{1}{2}}, \quad \sigma_{b b}=\frac{q}{j+\frac{1}{2}}, \quad \sigma_{a b}=-\sqrt{1-\left(\frac{q}{j+\frac{1}{2}}\right)^{2}},
$$

and of course $\sigma_{b a}=\sigma_{a b}^{*}=\sigma_{a b}$.

\section{B. Energy spectrum and Casimir energy for fermions on $\mathbf{S}^{2}$}

The energy spectrum can be read off from the behavior of the solutions as a function of $\tau$ : a solution with energy $E$ behaves as $e^{-E \tau}$. The results are as follows.

- Case (i): $q=0$.

The two equations decouple, and we find

$$
R^{a}(\tau)=C^{a} e^{\left(j+\frac{1}{2}\right) \tau}, \quad R^{b}(\tau)=C^{b} e^{-\left(j+\frac{1}{2}\right) \tau},
$$

where $C^{a}$ and $C^{b}$ are integration constants, and $j=\frac{1}{2}, \frac{3}{2}, \frac{5}{2} \ldots$ There are no zeroenergy solutions.

- Case (ii): $q \neq 0, j=|q|-\frac{1}{2}$.

In this case, there is no such thing as $R^{a}$ (because $l$ cannot be $j-\frac{1}{2}=|q|-1$ ). So the first equation is absent, and the second equation gives:

$$
R^{b}(\tau)=C
$$

with an arbitrary constant $C$. This solution has zero energy and degeneracy $2 j+1=$ $2|q|$.

- Case (iii): $q \neq 0$ and $j=|q|-\frac{1}{2}+p>|q|-\frac{1}{2}, p=1,2, \ldots$.

In this case, the two equations are coupled but easy to solve by eliminating one of the two unknowns. The result is

$$
\begin{aligned}
R^{a}(\tau)= & q C_{1} e^{\tau \sqrt{\left(j+\frac{1}{2}\right)^{2}-q^{2}}}+q C_{2} e^{-\tau \sqrt{\left(j+\frac{1}{2}\right)^{2}-q^{2}}} \\
R^{b}(\tau)= & {\left[\sqrt{\left(j+\frac{1}{2}\right)^{2}-q^{2}}-\left(j+\frac{1}{2}\right)\right] C_{1} e^{\tau \sqrt{\left(j+\frac{1}{2}\right)^{2}-q^{2}}}+} \\
+ & {\left[\sqrt{\left(j+\frac{1}{2}\right)^{2}-q^{2}}+\left(j+\frac{1}{2}\right)\right] C_{2} e^{-\tau \sqrt{\left(j+\frac{1}{2}\right)^{2}-q^{2}}}, }
\end{aligned}
$$


where $C_{1}$ and $C_{2}$ are integration constants. The corresponding energies are

$$
E_{p}= \pm \sqrt{\left(j+\frac{1}{2}\right)^{2}-q^{2}}= \pm \sqrt{2|q| p+p^{2}}
$$

with degeneracies $2 j+1=2|q|+2 p$. Note that the spectrum is symmetric under $q \rightarrow-q$.

The regularized Casimir energy is given by

$$
E_{r e g}(\beta)=-\sum_{p=0}^{\infty}(2 p+|n|) \sqrt{p^{2}+p|n|} e^{-\beta \sqrt{p^{2}+p|n|}} .
$$

We renormalize it by requiring that the Casimir energy of the vacuum with $n=0$ be zero. That is, we subtract from the above sum a similar sum with $n=0$, and then take the limit $\beta \rightarrow 0$. Using the Abel-Plana summation formula

$$
\sum_{p=0}^{\infty} F(p)=\frac{1}{2} F(0)+\int_{0}^{\infty} d x F(x)+i \int_{0}^{\infty} d t \frac{F(i t)-F(-i t)}{e^{2 \pi t}-1},
$$

we obtain a finite answer for the Casimir energy:

$$
\begin{aligned}
E_{\text {Casimir }}= & \frac{1}{6} \sqrt{1+|n|}(|n|-2)+ \\
& +4 \operatorname{Im} \int_{0}^{\infty} d t\left[\left(i t+\frac{|n|}{2}+1\right) \sqrt{\left(i t+\frac{|n|}{2}+1\right)^{2}-\frac{n^{2}}{4}}\right] \frac{1}{e^{2 \pi t}-1} .
\end{aligned}
$$

Here one needs to take the branch of the square root which is positive on the positive real axis. The integral cannot be expressed in terms of elementary functions, but can be easily evaluated numerically for any $n$.

\section{Acknowledgments}

This work grew out of attempts by one of the authors (A.K.) and M. J. Strassler to improve on the last section of ref. [11]. A.K. would like to thank M. J. Strassler for numerous discussions which helped to realize the importance of fermionic zero modes. We also would like to thank J. Maldacena, T. Okuda, and H. Ooguri for useful conversations, and S. Sachdev for informing us about ref. [28]. This work was supported in part by a DOE grant DE-FG03-92-ER40701.

\section{References}

[1] S.R. Coleman, Quantum sine-gordon equation as the massive thirring model, Phys. Rev. D $11(1975) 2088$.

[2] S. Mandelstam, Soliton operators for the quantized sine-gordon equation, Phys. Rev. D 11 (1975) 3026. 
[3] C. Montonen and D.I. Olive, Magnetic monopoles as gauge particles?, Phys. Lett. B 72 (1977) 117 .

[4] E. Witten and D.I. Olive, Supersymmetry algebras that include topological charges, Phys. Lett. B 78 (1978) 97.

[5] H. Osborn, Topological charges for $N=4$ supersymmetric gauge theories and monopoles of spin 1, Phys. Lett. B 83 (1979) 321.

[6] N. Seiberg, Electric - magnetic duality in supersymmetric nonabelian gauge theories, Nucl. Phys. B 435 (1995) 129 hep-th/9411149.

[7] M.E. Peskin, Duality in supersymmetric Yang-Mills theory, hep-th/9702094

[8] M. Chaichian, W.F. Chen and C. Montonen, New superconformal field theories in four dimensions and $N=1$ duality, Phys. Rept. 346 (2001) 89 hep-th/0007240.

[9] K.A. Intriligator and N. Seiberg, Mirror symmetry in three dimensional gauge theories, Phys. Lett. B 387 (1996) 513 hep-th/9607207.

[10] O. Aharony, A. Hanany, K.A. Intriligator, N. Seiberg and M.J. Strassler, Aspects of $N=2$ supersymmetric gauge theories in three dimensions, Nucl. Phys. B 499 (1997) 67 hep-th/9703110.

[11] A. Kapustin and M.J. Strassler, On mirror symmetry in three dimensional abelian gauge theories, J. High Energy Phys. 04 (1999) 021 hep-th/9902033.

[12] A.N. Redlich, Gauge noninvariance and parity nonconservation of three- dimensional fermions, Phys. Rev. Lett. 52 (1984) 18, Parity violation and gauge noninvariance of the effective gauge field action in three-dimensions, Phys. Rev. D 29 (1984) 2366.

[13] T. Appelquist and R.D. Pisarski, Hot Yang-Mills theories and three-dimensional QCD, Phys. Rev. D 23 (1981) 2305.

[14] R. Jackiw and S. Templeton, How superrenormalizable interactions cure their infrared divergences, Phys. Rev. D 23 (1981) 2291.

[15] S. Templeton, Summation of dominant coupling constant logarithms in qed in three-dimensions, Phys. Lett. B 103 (1981) 134, Summation of coupling constant logarithms in qed in three-dimensions, Phys. Rev. D 24 (1981) 3134.

[16] T. Appelquist and U.W. Heinz, Three-dimensional o(n) theories at large distances, Phys. Rev. D 24 (1981) 2169.

[17] T. Banks and A. Zaks, On the phase structure of vector - like gauge theories with massless fermions, Nucl. Phys. B 196 (1982) 189.

[18] R.D. Pisarski, Chiral symmetry breaking in three-dimensional electrodynamics, Phys. Rev. D 29 (1984) 2423.

[19] T. Appelquist, D. Nash and L.C.R. Wijewardhana, Critical behavior in $(2+1)$-dimensional qed, Phys. Rev. Lett. 60 (1988) 2575.

[20] D. Nash, Higher order corrections in (2+1)-dimensional qed, Phys. Rev. Lett. 62 (1989) 3024.

[21] P. Maris, The influence of the full vertex and vacuum polarization on the fermion propagator in qed3, Phys. Rev. D 54 (1996) 4049 [hep-ph/9606214. 
[22] M.R. Pennington and D. Walsh, Masses from nothing: a nonperturbative study of qed in three-dimensions, Phys. Lett. B 253 (1991) 246.

[23] D.C. Curtis, M.R. Pennington and D. Walsh, Dynamical mass generation in qed in three-dimensions and the 1/n expansion, Phys. Lett. B 295 (1992) 313.

[24] P. Ginsparg, Applied Conformal Field Theory, lectures given at Les Houches summer school in theoretical physics, Les Houches, France, Jun 28 - Aug 5, 1988

[25] R. Jackiw and C. Rebbi, Solitons with fermion number 1/2, Phys. Rev. D 13 (1976) 3398.

[26] A.J. Niemi and G.W. Semenoff, Axial anomaly induced fermion fractionization and effective gauge theory actions in odd dimensional space-times, Phys. Rev. Lett. 51 (1983) 2077.

[27] N. Seiberg and E. Witten, Monopoles, duality and chiral symmetry breaking in $N=2$ supersymmetric QCD, Nucl. Phys. B 431 (1994) 484 hep-th/9408099.

[28] G. Murthy and S. Sachdev, Action of hedgehog instantons in the disordered phase of the (2+1)-dimensional $C P^{(n-1)}$ model, Nucl. Phys. B 344 (1990) 557.

[29] T.T. Wu and C.N. Yang, Dirac monopole without strings: monopole harmonics, Nucl. Phys. B 107 (1976) 365. 